\title{
Localized accumulation of silicon (Si) in grape leaves affected by Pierce's disease.
}

\author{
B. Leite ${ }^{1} \&$ P. C. Andersen ${ }^{2}$ \\ ${ }^{1}$ Thermo Fisher Scientific, 2552 Verona Road, Madison, WI, 53711, USA.; \\ ${ }^{2}$ University of Florida, NFREC-IFAS, 155 Research Road, FL, 32351, USA.
}

The focus of this research is to analyze some possible chemical differences between healthy grapevine leaf areas and those exhibiting typical leaf scorch symptoms of Pierce's disease. This disease is caused by the pathogenic bacterium Xylella fastidiosa and has caused serious losses for the multi million grape and wine industry in California. Similar problems are also a concern for the Florida grape and wine industry. Several attempts are being made towards the development of strategies to confer resistance to plants against $X$. fastidios $a$. Field trials are being carried out with distinct approaches to stop the disease. However, there is no cure at this point for either Vitis vinifera (European) or Vitis labrusca (American) grapevines infected with Pierce's disease.

The successful disease development is dependent on several factors: a) the presence of an insect vector to spread the bacterium, b) the level of susceptibility of the grapevine, c) the capacity of the pathogen to multiply and establish a biofilm (community of cells) inside plant xylem vessels and d) the ability to transmit pathogens from plant to plant. Details of the disease mechanisms remain unresolved. Xylem vessels and xylem fluid chemistry is believed to play a crucial role the process, since bacterial cells survive, multiply and get established within this very unique environment. Xylem fluid is composed mainly of water and low concentrations of ions, amino acids and organic acids [ 1 ]. The hypothesis that plant vessel walls and bacterial surface chemistry is critical for Pierce's disease is an area of investigation [2].

In the present work, we have collected diseased grapevine leaves of $V$. labrusca growing in North Florida and we analyzed the tissue by Energy Dispersive Spectroscopy (EDS) utilizing a Silicon Drift Detector (SDD-30 $\mathrm{mm}^{2}$ ), under variable pressure (20-30 Pascal) and an accelerating voltage of $15 \mathrm{KeV}$. Results showed that leaf scorched areas (lesions) accumulated high concentrations of silicon (Si) (Fig. 1, Fig. 2). This metabolic transition is possibly a result of the plant strategy to cope with xylem vessel occlusion due to bacterial multiplication and the reduction of water flow. Si has the potential to significantly decrease the susceptibility of certain plants to both biotic and abiotic stress. It is known that Si-fertilized rice plants may even increase growth and yield, in addition to reducing disease severity [ 3 ]. We have used spectral imaging [ 4 ] to process the data cube and separate chemical phases (Fig. 3). This is the first work reporting Si accumulation in leaf scorched areas affected by Pierce's disease. Future investigations should reveal the influence of Si on the concentration and availability of calcium $(\mathrm{Ca})$ and magnesium $(\mathrm{Mg})$, as these elements may influence bacterial aggregation and adhesion.

References

[ 1 ] Andersen PC, Brodbeck BV. Physiol. Plant 1989;75:63-70.

[2 ] Leite et al, 2004. FEMS Microbiol. Lett. 2004;230:283-290.

[ 3 ] Danoff et al, 1997. Crop Protection. 16:525-531.

[ 4 ] Thermo Scientific, Noran System Seven. Spectral Imaging tools.

Acknowledgement: Dr. V. Colova (CVSFR-Florida A \& M Univ.) for providing the samples. 


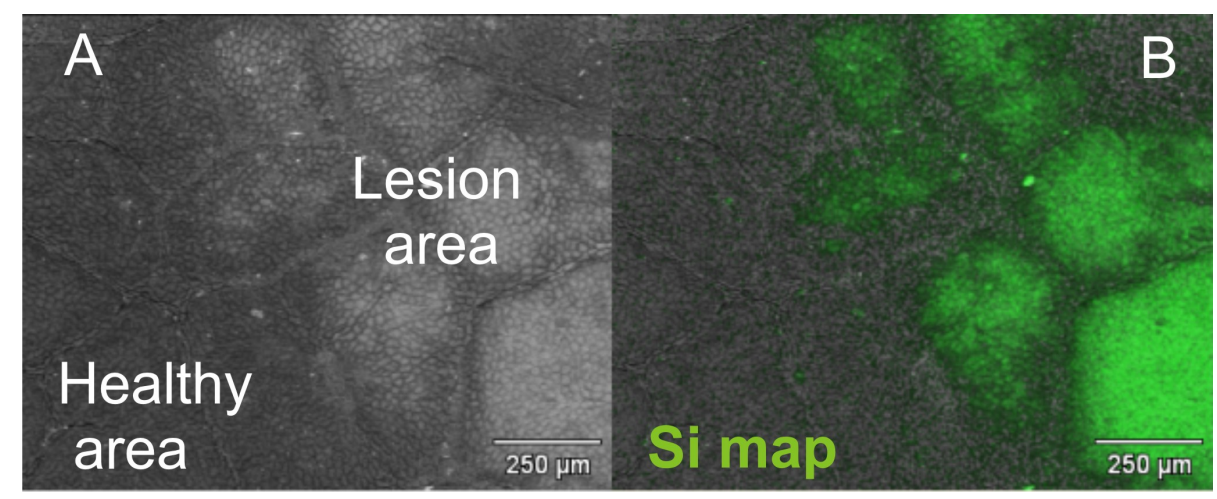

Fig 1 - X-Ray microanalysis was performed at the interface of a healthy area (control) and a Pierce's disease lesion area on a grapevine leaf. Notice that diseased (lesion) area accumulated silicon. Scanning Electron Microscope image (A), X-Ray silicon (Si) map (B).

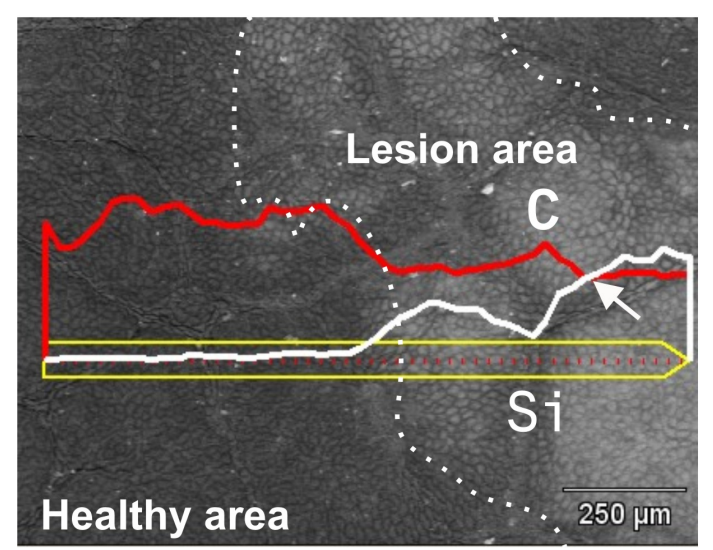

Fig 2 - Quantitative X-Ray line scan mapping (net counts) on areas of interest. Notice that carbon (C) is consistently high on healthy area and decreases as the line reaches the lesion area edge. In the lesion area the concentrations of $\mathrm{C}$ declines and $\mathrm{Si}$ increases.

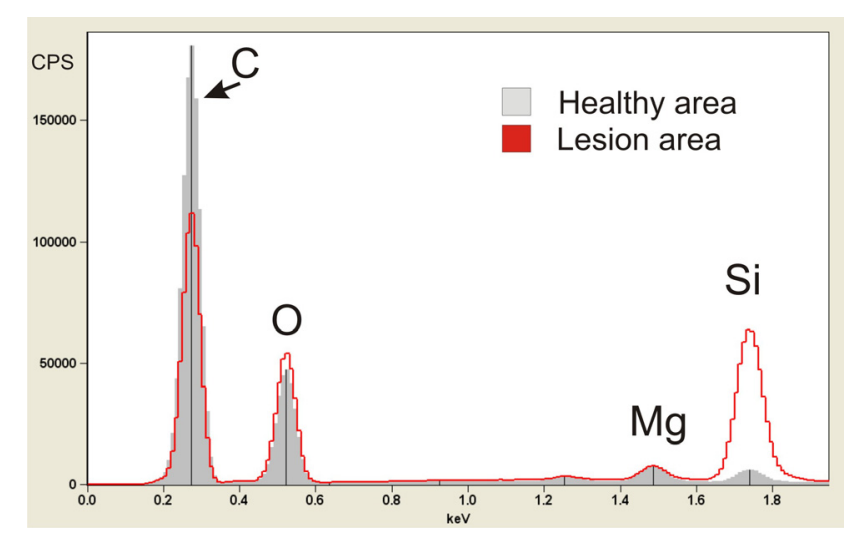

Fig 3 - Overlapped spectra of healthy and lesion areas after phase determination [ 4 ]. Notice differences in carbon (C) and silicon (Si) peak intensities. Further investigation is required to understand this shift mechanism. CPS $=$ X-Ray counts per second 